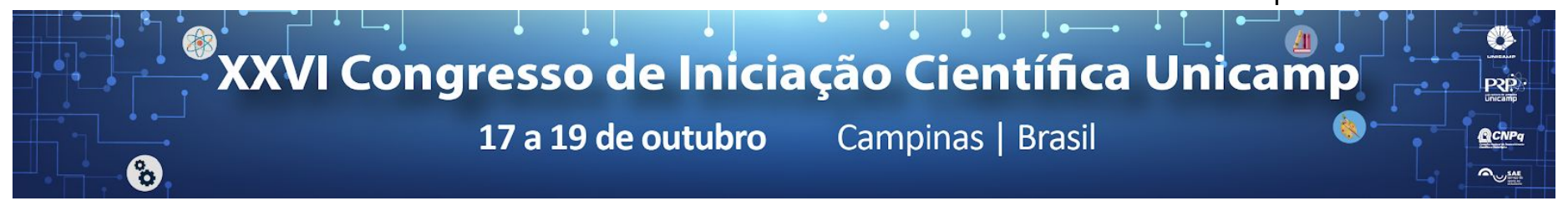

\title{
A Virtual Model of a Vascularized Bone Matrix for Biofabrication
}

\section{Maria B. Kersanach*, Janaina A. Dernowsek, Jorge V. L. Silva.}

\begin{abstract}
Increasingly, biofabrication is seen as a promising strategy in the tissue engineering and regenerative medicine fields. It proves to be a good alternative for drug and cosmetics testing and even for transplantation tissues and organs in humans. However, long before we dream with this science impacting our daily lives, we need to know it more on a smaller scale the cellular interactions involved, the biomolecules, the transcription factors, the differentiation phases - it is all highly correlated, sensitive and complex. With the aim of reducing the investments of large sums of money and time with in vitro experiments, this work proposes the creation of a predictive model to the biological structures that will be biofabricated. From the use of mathematical and computational methods, simulations of biological phenomena are made through the translation of the biological processes described in the literature into logical processes written in programming languages. These in silico strategies make possible to iteratively refine physical and biochemical parameters before the in vitro stage. To exemplify this approach, an osteogenesis and angiogenesis implementation is shown in a virtual tissue spheroid (the bioprinting basic unit) - from mesenchymal and endothelial cells to a vascularized bone matrix.
\end{abstract}

\section{Key words:}

Biofabrication, Osteogenesis, Computational Modeling

\section{Introduction}

A computational project of a biological structure can be achieved with a junction of different techniques - as treatment of medical images, computer-aided design, computer simulations, and artificial intelligence. In this project, attribute engineering is used to model the desired structure and make predictions of their possible behaviours. To do this, some key stimuli (as RUNX2, VEGF and Glucose) and cell phases (as mesenchymal, osteoblast, osteocyte and the final bone matrix) were selected and had their values varied and refined thinking of different applications. Thereby, after investigating the cell behaviour in this created model, the in vitro and in vivo experiments could be better understood - saving either time and resources of researchers in this field.

\section{Results and Discussion}

From the biological side, the spheroids technique was chosen because - for being a 3D structure - it mimics in a more reliable way the in vivo environment. The main process implemented was a couple of cell differentiation functional and structural cell changes looking for a specialization in the cell role.

In the computational side, the project was implemented on Compucell3D, an open-source software that implements the Glazier-Graner-Hogeweg (GGH) and the Monte Carlo Steps (MCS) modelling methods using $\mathrm{C}++$ and Python. The initial $X X X$ is a setup with two spheroids with $\sim 100 \mu \mathrm{m}$ diameter and $\sim 300$ cells (either mesenchymal and endothelial) who will form the vascularized bone matrix at the end of the process.

The main outcome of this work is the cells profile created through the modelling and simulating processes. It makes possible a better understanding of the functional and morphological changes during the tissues formation. In the image 1, for example, we can see some of these profiles since the spheroids formed with mesenchymal and endothelial cells (first line) to the vascularized bone matrix (last line). In this first phases of the project, the parameters choices were made applying reverse engineering in biological experiments from the literature.

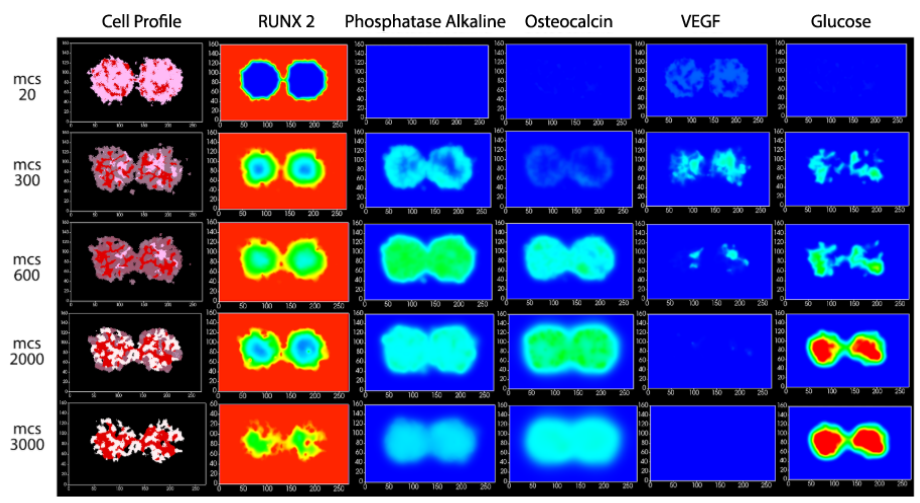

Image 1. Cells and biomolecules profiles during different steps of a bone matrix formation simulated in Compucell3D

\section{Conclusions}

The goal of this project was to use computational tools to better understand different cell behaviours, transforming the biofabrication into a more standardized process. The next steps will be integrating these results with other computational techniques aiming for a even more complete model.

\section{Acknowledgement}

The authors thank the support from the National Council for Scientific and Technological Development (CNPq) and the Institute of Computing Alumni Program (IC/UNICAMP).

Swat, M. H., et al. Methods in cell biology 2012, 110, 325-366

Orciani, M., et al. Frontiers in bioengineering and biotechnology 2017, 5, 17. 